\title{
Lien entre parodontopathies et prématurité : analyse critique de 15 ans de recherche
}

\author{
Mathieu Gunepin ${ }^{1, \star}$, Florence Derache ${ }^{2}$ \\ 1 Secteur dentaire inter-armées, Draguignan, France \\ 2 Service de Chirurgie maxillo-faciale et Stomatologie, HIA Sainte Anne, Toulon, France
}

(Reçu le 13 septembre 2010, accepté le 20 septembre 2010)

Mots clés :

parodontopathie / grossesse

/ accouchement prématuré

Key words:

periodontal disease /

pregnancy / preterm birth

\begin{abstract}
Résumé - En raison du taux d'incidence élevé, de la gravité des complications, de la lourdeur de la prise en charge et des possibilités en terme de prévention, les nouveau-nés prématurés représentent un des domaines d'intérêt pour la recherche médicale. La médecine bucco-dentaire prend part à ces travaux de recherche depuis 1996 en essayant de déterminer l'existence d'un lien entre les maladies parodontales et la prématurité.

Nous proposons ici une revue critique de la littérature internationale portant sur les travaux tendant à établir l'existence de ce lien, à la fois sur la forme (méthodologie) et sur le fond (conclusions).

Toute tentative de méta-analyse et d'élaboration de recommandations achoppe sur le caractère individuel de chaque étude car les critères utilisés pour définir la maladie parodontale et la prématurité sont différents ce qui conduit inéluctablement à des conclusions divergentes.

Cependant, en étudiant la bactériémie et l'inflammation spécifiques des maladies parodontales et leurs conséquences sur la grossesse, la biologie semble constituer un axe de recherche prometteur qui devrait permettre de préciser le lien entre maladie parodontale et prématurité.
\end{abstract}

Abstract - Periodontal diseases and preterm birth: a review of 15 years of medical research. The delivery of preterm babies (under 37 weeks $f$ gestation) is a significant public health issue. This problem is associated with both high postnatal mortality rate and higher risk of morbidity due to sequelae. Despite clinical researches, in $40 \%$ of cases, aetiology of preterm birth remains unknown and the preterm birth rates have not only not declined but also have increased over the last two decades (7\% in France, 13\% in the United States).

A potential link between odontology and preterm babies is studied for years. In 1996, some authors described periodontal disease as a risk factor of preterm birth. Since then, numerous studies were published to assert or deny this hypothesis. A classical meta-analysis of these works is quasi impossible due to huge methodological discrepancies between studies: definition and evaluation of periodontal disease are fluctuating, the criteria "low weight babies" is added to "preterm birth" by some authors, etc.

Even if an increasing of preterm birth rate was statistically proved in women with periodontal disease, it will not inevitably signify that periodontal disease is a risk factor of preterm birth, the events could be concomitant without a cause to effect link. So, nowadays, decreeing recommendations of oral care to pregnant women in order to prevent preterm birth based on so different studies and conclusions seems to be not reasonable.

However, biology seems to be a promising area of research which should clarify the link between periodontal disease and preterm birth in the next few years. The suggested mechanisms need to be proved based on studies of the concentrations and impact on pregnancy of some molecules produced due to periodontal disease.

\footnotetext{
*Correspondance : mgunepin@yahoo.fr
} 


$\begin{array}{ll}\text { Abréviations } & \\ \text { AG } & \text { : âge gestationnel } \\ \text { CI } & \begin{array}{l}\text { indice de tartre } \\ \text { : indice communautaire des besoins } \\ \text { CPITN }\end{array} \\ & : \text { indice gingival } \\ \text { GI } & : \text { interleukine } \\ \text { IL } & \text { lipopolysaccharide } \\ \text { LPS } & : \text { prostaglandine } \\ \text { PG } & : \text { indice de plaque } \\ \text { PI } & \text { gingival papillae (P), buccal or labial } \\ \text { PMA } & \text { gingival margin (M), attached } \\ & \text { gingiva (A) } \\ \text { PTNS } & \text { de traitement } \\ & : \text { semaines d'aménorrhée } \\ \text { SA } & : \text { indice de saignement parodontal } \\ \text { SBI } & \text { tumor necrosis factor }\end{array}$

La prématurité et la maladie parodontale sont des phénomènes étudiés depuis deux décennies. De ce fait, leur définition, fournie par des entités institutionnelles nationales et internationales (Haute Autorité de Santé, Organisation Mondiale de la Santé, etc.), devrait faire consensus. Cependant une revue de la littérature internationale portant sur l'évaluation du lien entre prématurité et maladie parodontale montre qu'il n'en est rien. Des études épidémiologiques qui semblent comparables portent en fait sur des éléments très différents : l'acquisition et l'analyse des données nécessaires à l'établissement de l'existence d'un lien s'avèrent très délicates dans ce contexte multifactoriel extrêmement complexe. L'impact de la méthodologie sur les résultats des études ne pouvant être évalué, il est difficile, voire impossible d'interpréter les divergences entre les conclusions des auteurs. C'est pourquoi on cherche à exploiter d'autres axes de recherche en dehors du champs épidémiologique (approches thérapeutique et biologique) mais les résultats sont également inégaux.

\section{Prématurité}

\section{Définition}

La prématurité est une naissance survenant avant 37 semaines révolues d'aménorrhée, c'est-à-dire avant le 259 ème jour calculé à partir du premier jour des dernières règles, quel que soit le poids de l'enfant à la naissance [1]. L'âge gestationnel (AG) du prématuré peut être connu à l'aide de la date des dernières règles (ce renseignement peut être imprécis), par une échographie réalisée avant la $12^{\text {ème }}$ semaine d'aménorrhée (SA) ou par l'examen clinique du prématuré.

L'incidence de la prématurité est de 6 à $7 \%$ en France, soit 40000 prématurés par an [2], de 11 à $13 \%$ aux EtatsUnis $[3,4]$ et de 5 à $9 \%$ dans l'ensemble des autres pays développés $[3,5]$. Le taux de survie dépend de l'AG, la zone frontière se situant à $32 \mathrm{SA}$.

\section{Étiologies}

Les causes de la prématurité ne sont retrouvées que dans $60 \%$ des cas et $30 \%$ des accouchements prématurés font suite à une décision médicale lorsque les risques liés à la poursuite de la grossesse sont supérieurs à ceux liés à la prématurité.

Les causes les plus fréquentes sont $[6,7]$ :

- obstétricales :

- grossesses multiples (10 à $25 \%$ des menaces d'accouchement prématuré)

- placenta prævia

- béance cervico-isthmique

- malformations utérines

- rupture de la poche des eaux

- maternelles :

- infections (générale, urinaire, cervico-vaginale)

- diabète

- toxémie gravidique

- immunisation rhésus

- fœtales :

- retard de croissance intra-utérin

- malformations et anomalies chromosomiques

D'autres facteurs favorisants sont connus [1,8-14] :

- âge de la mère (plus de 35 ans ou moins de 18 ans)

- tabagisme, alcoolisme, toxicomanie

- multiparité, primiparité

- antécédents obstétricaux

- antécédents d'interruption volontaire de grossesse

- conditions de travail ou de vie stressantes

- grossesses particulièrement rapprochées

- mauvaises conditions socio-économiques, déplacements répétés, position debout prolongée...

\section{Parodontopathies}

Les maladies parodontales ou parodontopathies peuvent être définies comme des maladies infectieuses multifactorielles. Elles sont caractérisées par des symptômes et des signes cliniques qui peuvent inclure une inflammation visible ou non, des saignements gingivaux spontanés ou provoqués d'importance variable, la formation de poches parodontales en rapport avec la perte de l'attache gingivale et de l'os alvéolaire, et une mobilité dentaire, elles peuvent conduire à la perte de dents [15].

La classification des maladies parodontales est complexe. Celle proposée par l'American Association of Periodontology [16] est basée sur les symptômes cliniques, le type de tissus atteints, l'étendue et le degré de ces atteintes tissulaires, avec un âge limite de 35 ans qui détermine le passage de la parodontite agressive à la parodontite chronique à un stade avancé. Elle prend en compte l'impact de maladies générales (maladies systémiques, maladies génétiques, hémopathies...) sur le parodonte, et distingue les gingivites causées 
par la plaque bactérienne et celles qui ne sont pas causées par la plaque bactérienne [16].

Des indices cliniques et/ou épidémiologiques spécifiques ont été définis pour évaluer le degré d'inflammation, la présence de plaque, la présence de tartre, le niveau de l'attache clinique, la mesure de la profondeur des poches. Ce sont principalement [15] :

- les indices d'hygiène : l'indice d'hygiène buccale de Greene et Vermillion, l'indice de plaque de Silness et Löe (PI), l'indice de plaque de 0 'Leary, l'indice de tartre de Marthaler (CI) ;

- les indices d'inflammation : l'indice gingival de Löe et Silness (GI), l'indice de saignement parodontal (SBI), l'indice PMA de Schour-Massler;

- les indices de besoins de traitement : l'indice parodontal des besoins de traitement (PTNS), l'indice communautaire des besoins en soins parodontaux (CPITN); cet indice est actuellement utilisé pour les enquêtes épidémiologiques, les projets de santé publique et la promotion de la santé parodontale.

\section{Revue de la littérature sur le lien entre parodontopathies et prématurité}

La première étude établissant un lien entre parodontopathies et prématurité a été publiée en 1996 par Offenbacher et al. [17]. L'intérêt suscité par cette étude au sein de la communauté scientifique est lié à l'importance du facteur liant ces deux phénomènes : les femmes souffrant de parodontopathies auraient 7,9 fois plus de risque d'accoucher prématurément que les autres femmes. De nombreux articles ont été publiés pour essayer de confirmer ou d'infirmer cette assertion. Une revue de la littérature internationale sur ce lien hypothétique met en évidence de nombreuses divergences dans les études sur la forme (méthodologie et terminologie) et sur le fond (conclusion).

\section{Définition de la prématurité}

En ce qui concerne le nouveau-né, de nombreux auteurs parlent de «prématurés de faible poids à la naissance (moins de $2500 \mathrm{~g}$ ) » ce qui aboutit à confondre l'hypotrophie et la prématurité [17-24]. Ceci biaise les statistiques puisque si l'on additionne les prématurés et les nouveau-nés hypotrophiques, on obtient un nombre supérieur à celui des « prématurés de faible poids » car certains nouveau-nés sont comptabilisés dans les deux catégories (de nombreux prématurés sont de faible poids mais pas tous).

Certaines études prennent en compte l'impact supposé de la maladie parodontale sur :

- le poids du nouveau-né [25-34];

- la prématurité telle qu'elle a été définie précédemment (accouchement avant 37 SA quel que soit le poids du nouveau-né) [29-40] ;
- le poids et la prématurité $[29-33,41]$;

- le poids ou la prématurité [31,42-44].

De plus, le calcul de l'AG n'est pas toujours évident, or peu d'auteurs indiquent leurs méthodes de calcul de l'AG. Ces méthodes sont cliniques et/ou échographiques et/ou basées sur le dernier cycle menstruel. Les problèmes liés à l'utilisation du dernier cycle menstruel sont nombreux : menstruations irrégulières, utilisation de contraceptifs oraux, saignements durant le premier trimestre de la grossesse pouvant fausser le calcul de l'AG. La fréquence des nouveau-nés dits prématurés est d'ailleurs plus importante lorsque cette technique est utilisée : ceci se traduit par une surestimation de la fréquence de prématurité. Les résultats obtenus par échographie sont aléatoires après 18 semaines de grossesse, ce qui peut une nouvelle fois fausser la fréquence des prématurés. Pour préciser le terme avec une faible marge d'erreur, une échographie précoce doit être réalisée (avant la $12^{\text {ème }} \mathrm{SA}$ ).

Le critère "conséquence sur la grossesse » diffère donc largement d'une étude à l'autre et les méthodes de calcul de l'AG non décrites ou potentiellement biaisées empêchent toute comparaison entre les études.

\section{Définition et évaluation de la maladie parodontale}

Après cette revue de la littérature, on doit constater que la définition de la maladie parodontale varie selon les auteurs. Les mesures pour évaluer l'atteinte parodontale varient également d'une étude à l'autre. Certains auteurs utilisent le CPITN qui est considéré comme non adapté pour l'évaluation de la sévérité et de la prévalence de la maladie parodontale dans les études cliniques [45]. Le CPITN donne une évaluation globale alors que la classification des parodontopathies est complexe et précise. Sont également utilisés afin de caractériser la maladie parodontale : la perte d'attache clinique, le PI, le GI, les analyses microbiologiques, la mobilité dentaire, etc. [7]. Certains auteurs utilisent un seul de ces paramètres [46], voire deux $[20,37,47,48]$ alors que d'autres en utilisent jusqu'à six [49-51]. Ceci représente un obstacle pour la réalisation des méta-analyses car la définition de la maladie parodontale n'est pas comparable d'une étude à l'autre [7].

De plus, il est difficile d'évaluer la fréquence de la maladie parodontale. En englobant toutes les manifestations de la maladie parodontale (gingivite, parodontite, etc.), $90 \%$ des individus seraient atteints [52], donc aussi les femmes enceintes. La fréquence des individus, au sein de la population générale, ayant des poches parodontales supérieures ou égales à $4 \mathrm{~mm}$, varie selon les études de 10 à $69 \%$. La fréquence des gingivites gravidiques varie quant à elle de 35 à $100 \%$ selon les auteurs [53] et la fréquence des autres formes de gingivites de 30 à $100 \%$ [54]. Ces importantes variations traduisent la « lecture » extrêmement individuelle et praticien-dépendant de la maladie parodontale qui reste difficile à objectiver et à quantifier. 


\section{Prise en compte des cofacteurs}

De nombreuses causes peuvent être à l'origine de la prématurité et plusieurs facteurs favorisent sa survenue. Dans la revue de littérature de Vettore et al., parue en 2006, on constate que peu d'études prennent en compte les facteurs de risque [45]. Dans la méta-analyse réalisée par Vergnes et al. [7], les 17 études analysées prennent en compte de 6 à 14 facteurs de risque sur les 43 décrits par Vettore et al. [45]. Des auteurs excluent de leur échantillon certaines femmes $[20,31,40]$ (patientes ayant un diabète, une maladie cardiovasculaire, un asthme, etc.) ce qui rend difficile la réalisation de toute méta-analyse car, les échantillons n'étant pas similaires, on ne peut pas comparer les résultats concernant l'incidence des prématurités. Plusieurs auteurs ont souligné que cette absence de prise en compte de l'ensemble des co-facteurs constitue un biais majeur qui conduit à relativiser les résultats des études réalisées $[7,45]$.

\section{Revue critique des conclusions des différentes recherches}

En raison de l'importance du sujet, de nombreux articles et recherches ont porté sur le lien entre prématurité et parodontopathies au cours des 15 dernières années. Lorsque les données sont devenues suffisamment abondantes, on a vu apparaitre les premières revues de la littérature $[45,55,56]$. Toutefois, avec de l'hétérogénéité des études, des paramètres étudiés, etc., la réalisation de méta-analyses s'avère extrêmement complexe [7], voire impossible pour certains auteurs $[45,57]$.

Deux éléments prégnants ressortent de ces travaux :

- Les études aboutissent à des conclusions divergentes. En ce qui concerne les revues de la littérature, Vettore et al. notent que, sur les 36 études qu'ils ont sélectionnées dans leur revue de la littérature, 26 concluaient à une association entre les deux phénomènes [45], Clothier et al. 22 études sur 31 [58] et Xiong et al. 18 sur 25 [59]. Cependant, bien que le bilan apparaisse plutôt favorable à l'existence d'un lien entre maladie parodontale et prématurité, de nombreuses études appliquant une méthodologie correcte concluent à une absence de lien [32,60-66]. Pour les études concluant à l'existence d'un lien, celui-ci peut être fort $[17,67]$ ou faible [68].

- Presque tous les auteurs s'accordent pour dire que d'autres recherches doivent être entreprises pour arriver à une conclusion fiable, ce qui suggère que les études actuelles sont insuffisantes $[7,56]$. C'est notamment la conclusion d'une revue de la littérature publiée en septembre 2008 et basée sur 12 ans de recherche [56].

\section{Approche thérapeutique}

Devant les difficultés méthodologiques rencontrées avec les études épidémiologiques, certains auteurs ont proposé un autre axe de recherche : essayer d'évaluer l'effet du traitement de la maladie parodontale sur la fréquence des prématurités $[55,61]$. Mais une fois encore, les réponses divergent: certains auteurs affirment que ces traitements sont efficaces pour prévenir la prématurité [69-75] alors que d'autres arrivent à une conclusion opposée [19,60,61,76-78]. Ainsi, Polyzos et al. considèrent que les traitements parodontaux réduisent significativement l'incidence des prématurités [70] tandis que Stamilio et al., dans un éditorial publié dans l'American Journal of Obstetrics and Gynecology, concluent que, sur la base des données actuelles, les traitements parodontaux ne peuvent pas être recommandés pour la prévention de la prématurité [79]. Macones et al. signalent même que les traitements parodontaux pourraient être à l'origine de certaines formes de prématurité [77].

Là aussi, ces divergences ne sont pas en rapport avec la qualité des études mais elles sont liées à la difficulté de réaliser ce type d'étude. En effet, le praticien ne dispose pas du temps nécessaire pour réaliser le traitement de la maladie parodontale pendant la grossesse (période relativement courte, impératifs d'abstention thérapeutique durant certaines phases de la grossesse, etc.).

\section{Approche biologique}

Ne pouvant pas prouver statistiquement la réalité de l'impact de la maladie parodontale et de son traitement sur la prématurité, la recherche s'est orientée vers la biologie, c'està-dire l'étude des mécanismes liant la maladie parodontale à la prématurité. Au cours de la grossesse, les infections de la cavité buccale, comme les parodontopathies, représentent une source d'inflammation et d'infection. On fait l'hypothèse que le processus inflammatoire et les bactériémies seraient à l'origine de prématurités. Ce dernier axe de recherche semble prometteur [21, 25, 36, 39, 80, 81].

La maladie parodontale est une infection à Gram négatif. Pendant le second semestre de la grossesse, la proportion de bactéries anaérobies Gram négatif par rapport aux bactéries aérobies Gram positif augmente dans la plaque dentaire [82]. Ces bactéries Gram négatif, associées à une pathologie telle que la maladie parodontale, peuvent produire une variété de molécules pouvant affecter l'hôte. Un composant microbien, le LPS, peut activer les macrophages et d'autres cellules pour synthétiser et secréter un large spectre de molécules comme les cytokines IL-1 $\beta$, TNF- $\alpha$, IL- 6 et PGE2 $[82,83]$. Si ces composants passent dans la circulation sanguine et traversent la barrière placentaire, les concentrations de PGE2 et de TNF$\alpha$ augmentent alors dans le liquide amniotique. Ceci pourrait être à l'origine de la prématurité [82].

L'analyse du cordon ombilical de 351 nouveau-nés a mis en évidence, chez les prématurés, une concentration en IgM antigermes spécifiques des pathologies buccales beaucoup plus élevée que chez les autres nouveau-nés [35]. Comme les IgM maternelles ne traversent pas la barrière placentaire, ceci signifierait une exposition intra-utérine directe du fœtus à ces bactéries, lesquelles pourraient être responsables de la prématurité. 
Le risque de prématurité semble augmenter lorsque le foetus est exposé à des bactéries d'origine parodontale [84]. Ces conclusions font suite à l'analyse des concentrations en protéine $C$ réactive, IL-1 $\beta$, TNF- $\alpha$, IL- 6 et PGE2, etc. dans le sang de 640 cordons ombilicaux de nouveau-nés.

\section{Discussion}

De façon empirique, les praticiens sont portés à croire à l'existence d'un lien entre maladie parodontale et prématurité mais, à l'ère de l'evidence-based medicine, les preuves font défaut. Les études épidémiologiques sont complexes (taille des échantillons, nombre de cofacteurs, définition de la maladie parodontale, etc.) et l'approche thérapeutique délicate car la période durant laquelle la femme peut être traitée est courte. L'axe de recherche le plus prometteur semble être actuellement la biologie. Le décryptage des mécanismes associant la maladie parodontale à la prématurité sur la base de l'étude de la concentration de certaines molécules et de leurs effets permettra sans doute d'obtenir une réponse scientifique sur l'existence ou non d'un lien entre les deux phénomènes.

Malgré l'absence de preuves scientifiques établissant un lien entre maladie parodontale et prématurité, des guides de bonnes pratiques sont d'ores et déjà édictés. En France par exemple, la Haute Autorité de Santé (HAS) indique que «les études disponibles ne permettent pas de conclure avec certitude à l'existence d'un lien de causalité entre maladie parodontale et accouchement prématuré. Néanmoins, le groupe de travail recommande une surveillance clinique particulière des femmes enceintes » [15]. Ainsi, la découverte d'une parodontite chez une femme enceinte nécessite sa prise en charge et une surveillance obstétricale accrue car la maladie parodontale semble, selon l'HAS, significativement associée à un risque de prématurité et de petit poids à la naissance. Cette assertion est une recommandation de niveau $C$, c'est-à-dire fondée sur des études de faible niveau de preuve. Aux EtatsUnis, l'usage du conditionnel est la règle. L'American Dental Association, l'Academy of Pediatric Dentistry et l'American Academy of Periodontology indiquent que l'amélioration de la santé bucco-dentaire des femmes enceintes pourrait réduire l'incidence des accouchements prématurés et des naissances de nouveau-nés de faible poids à la naissance $[76,85]$. L'American Medical Association précise que, dans ce contexte, les femmes devraient consulter un chirurgien dentiste dès le début de leur grossesse ou même dès lors qu'elles souhaitent être enceintes afin de dépister l'ensemble des pathologies bucco-dentaires et notamment les pathologies parodontales [86]. L'« Oral Health care during pregnancy and early childhood - practice guidelines » publié en 2006 souligne toutefois que la revue de la littérature ne permet pas d'établir un consensus ou un lien de causalité [87]. Au Canada, les informations diffusées auprès du grand public sont plutôt affirmatives : «les femmes souffrant de maladies des gencives courent plus de risques de mettre au monde un enfant prématuré ou de petit poids » [88]. Certains auteurs affirment même que, par «extrapolation statistique », 18,2 \% des 250000 naissances de nouveau-nés prématurés ou de faible poids à la naissance sont attribuables à une infection parodontale [89].

Les guides de bonnes pratiques et les recommandations semblent traduire un principe de précaution : si la maladie parodontale peut favoriser les accouchements prématurés, autant la prévenir et la traiter. Même si le lien n'est pas scientifiquement établi, les prématurités sont tellement fréquentes, avec des conséquences délétères et une étiologie très souvent inconnue, qu'aucune cause même hypothétique ne peut être écartée. Ce qui est certain, c'est que la prévention et le traitement la maladie parodontale ne peuvent être que recommandés pour toute femme enceinte ou non, puisqu'ils participent à la santé bucco-dentaire [90-93].

\section{Conclusion}

Dans le domaine de la médecine bucco-dentaire, peu de sujets font autant l'objet de controverses entre les chercheurs que l'établissement de l'existence d'un lien de causalité entre parodontopathies et prématurité. Alors que, de façon empirique, la plupart des praticiens sont convaincus de l'existence de ce lien, une revue de la littérature internationale montre que certains auteurs pensent avoir démontré son existence, d'autres son inexistence et quelques autres sont dubitatifs.

Les divergences de méthodologie sont flagrantes, notamment dans l'acquisition des données (techniques de calcul de l'AG, méthodes d'évaluation de la maladie parodontale...). Ces divergences méthodologiques ne sont pas en rapport avec la qualité des travaux mais simplement le reflet des difficultés rencontrées pour mesurer les paramètres, constituer des échantillons et prendre en compte un grand nombre de cofacteurs. L'exploitation des données de la littérature est donc difficile et il est scientifiquement impossible de conclure à l'existence irréfutable d'un lien de causalité.

L'étude de l'impact des traitements parodontaux sur la fréquence des prématurités n'est guère satisfaisante. La durée nécessaire à la réalisation des traitements parodontaux est peu compatible avec la période très courte durant laquelle le praticien peut intervenir au cours de la grossesse. Pour pallier ces difficultés, un nouvel axe de recherche semble se dessiner avec le recours à la biologie. La relation entre les évènements biologiques liés à la maladie parodontale et l'accouchement prématuré fait l'objet d'études qui apparaissent prometteuses. Ces travaux permettent d'envisager une intervention ciblée pour interrompre cette cascade de réactions biologiques qui aboutit à l'accouchement prématuré. Si on obtenait ainsi une diminution statistiquement significative du taux de prématurité chez les femmes traitées, le lien entre parodontopathies et prématurité serait ainsi clarifié.

Dans l'attente de ce progrès, le suivi parodontal des femmes enceintes ne peut être que recommandé comme il l'est pour l'ensemble de la population afin de garantir une bonne santé bucco-dentaire.

Conflits d'intérêt : aucun 


\section{Références}

1. Foix-L'Hélias L, Ancel PY, Blondel B. Facteurs de risque de prématurité en France et comparaison entre prématurité spontanée et prématurité induite. Résultats de l'enquête nationale périnatale de 1995. J Gynecol Obstet Biol Reprod 2000;29:55-65.

2. Meyer R, Lévy J, Melchior J. Précis d'obstétrique, $6^{\mathrm{e}}$ ed. Masson, Paris, 2001.

3. Goldenberg RL, Culhane JF, Iams JD, Romero R. Epidemiology and causes of preterm birth. Lancet 2008;371:75-84.

4. Martin JA, Kochanek KD, Strobino DM, Guyer B, MacDorman MF. Annual summary of vital statistics - 2003. Pediatrics 2005;115:619-34.

5. Palla's Alonso CR. Bustos Lozano G, Grupo PREVINFAD. El nino nacido prematuro. Recomendaciones. Rev Pediat Atencion Primaria 2003;5:45-72.

6. Goldenberg RL, Culhane JF. Prepregnancy health status and the risk of preterm delivery. Arch Pediatr Adolesc Med 2005;159:8990.

7. Vergnes JN, Sixou M. Preterm low birth weight and maternal periodontal status: a meta-analysis. Am J Obstet Gynecol 2007;196:135.e1-7.

8. Andres RL, Day MC. Perinatal complications associated with maternal tobacco use. Semin Neonatol 2005;5:231-41.

9. Smith GC, Pell JP, Dobbie R. Interpregnancy interval and risk of preterm birth and neonatal death: retrospective cohort study $\mathrm{Br}$ Med J, 2003;327:13.

10. Saurel-Cubizolles MJ, Zeitlin J, Lelong N, Papiernik E, Di Renzo GC, Bréart G; Europop Group. Employment, working conditions, and preterm birth: results from the Europop case-control survey. J Epidemiol Community Health 2004;58:395-401.

11. Pompeii LA, Savitz DA, Evenson KR, Rogers B, McMahon M. Physical exertion at work and the risk of preterm delivery and small-for-gestational-age birth. Obstet Gynecol 2005;106:127988.

12. Hendler I, Goldenberg RL, Mercer BM, Iams JD, Meis PJ, Moawad AH, MacPherson CA, Caritis SN, Miodovnik M, Menard KM, Thurnau GR, Sorokin Y. The preterm prediction study: association between maternal body mass index (BMI) and spontaneous and indicated preterm birth. Am J Obstet Gynecol 2005;192:882-6.

13. Li D, Liu L, Odouli R. Presence of depressive symptoms during early pregnancy and the risk of preterm delivery: a prospective cohort study. Hum Reprod 2009;24:146-53. Epub 2008 0ct 23.

14. Mercer BM, Goldenberg RL, Moawad AH, Meis PJ, Iams JD, Das AF, Caritis SN, Miodovnik M, Menard MK, Thurnau GR, Dombrowski MP, Roberts JM, McNellis D. The preterm prediction study: effect of gestational age and cause of preterm birth on subsequent obstetric outcome. National Institute of Child Health and Human Development Maternal-Fetal Medicine Units Network. Am J Obstet Gynecol 1999;181:1216-21.

15. Haute Autorité de Santé. Parodontopathies : diagnostics et traitements. Service des recommandations et références professionnelles (mai 2002).

16. Armitage GC. Development of a classification system for periodontal diseases and conditions. Ann Periodontol 1999;4:1-6.

17. Offenbacher S, Katz V, Fertik G, Collins J, Boyd D, Maynor G, McKaig R, Beck J. Periodontal infection as a possible risk factor for preterm low birth weight. J Periodontol 1996;67(Suppl.):1103-13.
18. Offenbacher S, Jared HL, O'Reilly PG, Wells SR, Salvi GE, Lawrence HP, Socransky SS, Beck JD. Potential pathogenic mechanisms of periodontitis associated pregnancy complications. Ann Periodontol 1998;3:233-50.

19. Davenport ES, Williams CE, Sterne JA, Murad S, Sivapathasundram V, Curtis MA. Maternal periodontal disease and preterm low birth weight: case-control study. J Dent Res 2002;81,313-8.

20. Moliterno LF, Monteiro B, Figuero CMS, Fischer RG. Association between periodontitis and low birth weight : a case control study. J Clin Periodontol 2005;32:886-90.

21. Konopka T, Rutkowska M, Hirnle L, Kopec W, Karolewska E. The secretion of prostaglandin E2 and interleukin 1-beta in women with periodontal diseases and preterm low-birth-weight. Bull Grup Int Rech Sci Stomatol Odontol 2003;45:18-28.

22. Carta G, Persia G, Falciglia K, Iovenitti P. Periodontal disease and poor obstetrical outcome. Clin Exp Obstet Gynecol 2004;31:479.

23. Noack B, Klingenberg J, Weigelt J, Hoffmann T. Periodontal status and preterm low birth weight: a case control study. J Periodontal Res 2005;40:339-45.

24. Buduneli N, baylas $H$, Buduneli E, Turkoglu 0, Kose T, Dahlen G. Periodontal infections and preterm low birth weight: a case control study. J Clin Periodontol 2005;32:174-81.

25. Dasanayake, AP, Boyd D, Madianos PN, Offenbacher S, Hills E. The association between Porphyromonas gingivalis-specific maternal serum IgG and low birth weight. J Periodontol 2001;72:1491-7.

26. Dasanayake AP. Poor periodontal health of the pregnant woman as a risk factor for low birth weight. Ann Periodontol 1998;3:206-12.

27. Sembene M, Moreau JC, Mbaye MM, Diallo A, Diallo PD, Ngom $M$, Benoist HM. Periodontal infection in pregnant women and low birth weight babies. Odontostomatol Trop 2000;89:19-22.

28. Louro PM, Fiori HH, Louro P, Steibel J, Fiori RM. Periodontal disease in pregnancy and low birth weight. J Pediatr (Rio J) 2001;77:23-8.

29. Moore S, Ide M, Coward PY, Randhawa M, Borkowska E, Baylis R, Wilson RF. A prospective study to investigate the relationship between periodontal disease and adverse pregnancy outcome. Br Dent J 2004;197:251-8.

30. Jarjoura K, Devine PC, Perez-Delboy A, Herrera-Abreu M, D'Alton M, Papapanou PN. Markers of periodontal infection and preterm birth. Am J Obst Gynecol 2005;192:513-9.

31. Lunardelli AN, Peres MA. Is there an association between periodontal disease, prematurity and low birth weight ? A population-based study. J Clin Periodontol 2005;32:938-46.

32. Moreu G, Tellez L, Gonzalez-Jaranay M. Relationship between maternal periodontal disease and low birth weight pre-term infants. J Clin Periodontol 2005;32:622-7.

33. Cruz SS, Costa MC, Filho IS, Vianna MI, Santo CT. Maternal periodontal disease as a factor associated with low birth weight. Rev Saùde Pùblica 2005;39:782-7.

34. Shirmohammadi A, Pourabbas R,Bilan N. Relationship between maternal periodontal condition and body size of newborns. Iran J Pediatr 2009;19:101-7.

35. Madianos PN, Lieff S, Murtha AP, Boggess KA, Auten RL Jr, Beck JD, Offenbacher S. Maternal periodontitis and prematurity. Part II: Maternal infection and fetal exposure. Ann Periodontol 2001;6:175-82 
36. Hasegawa K, Furuichi Y, Shimotsu A, Nakamura M, Yoshinaga M, Kamitomo M, Hatae M, Maruyama I, Izumi Y. Associations between systemic status, periodontal status, serum cytokine levels, and delivery outcomes in pregnant women with a diagnosis of threatened premature labor. J Periodontol 2003;74:1764-70.

37. Goepfert AR, Jeffcoat MK, Andrews WW, Faye-Petersen 0. Periodontal disease and upper genital tract inflammation in early spontaneous preterm birth. Obstet Gynecol 2004;104:7783.

38. Moore S, Randhawa M, Ide M. A case-control study to investigate an association between adverse pregnancy outcome and periodontal disease. J Clin Periodontol 2005;32:1-5.

39. Moore S, Ide M, Randawha M, Walker JJ, Reid JG, Simpson NA. An investigation into the association among preterm birth, cytokine gene polymorphisms and periodontal disease. BJOG 2004;111:125-32.

40. Glesse S, Saba-Chujfi E, Carvalho A, Pacheco JJ, Salazar F. Estudo epidemiológico da influência da doença periodontal no trabalho de parto pré-termo na cidade brasileira de Santa Cruz do Sul RS. Rev Port Estomatol Chir Maxilofac 2004;45:205-14.

41. Lopez NJ, Smith PC, Gutierrez J. Higher risk of preterm birth and low birth weight in women with periodontal disease. J Dent Res 2002;81:58-63.

42. Mitchell-Lewis D, Engebretson SP, Chen J, Lamster IB, Papapanou PN. Periodontal infections and pre-term birth: early findings from a cohort of young minority women in New York. Eur J Oral Sci 2001;109:34-9.

43. Mokeem SA, Molla GN, Al-Jewair TS. The prevalence and relationship between periodontal disease and pre-term low birth weight infants at King Khalid University Hospital in Riyad, Saudi Arabia. J Contemp Dent Pract 2004;5;40-56.

44. Dörtbudak 0, Eberthardt R, Ulm M, Persson RG. Periodontitis, a marker of risk in pregnancy for preterm birth. J Clin Periodontol 2005;32:45-52.

45. Vettore MV, de Almeida Lamarac G, Leao AT, Thomaz FB, Do Carom Leal M. Periodontal infection and adverse pregnancy outcomes: a systematic review of epidemiological studies. Cad saude Publica 2006;22:2041-53.

46. Louro PM, Fiori HH, Filho PL, Steibel J, Fiori RM. Periodontal disease in pregnancy and low birth weight. J Pediatr (Rio J) 2001;77:23-8.

47. Guimares AN, Silva-Mato A, Miranda Cota LO, Siqueira FM, Costa F0. Maternal periodontal disease and preterm or extreme preterm birth: an ordinal logistic regression analysis. J Periodontol 2010;81:350-8.

48. Jeffcoat MK, Geurs NC, Reddy MS, Cliver SP, Goldenberg RL, Hauth JC. Periodontal infection and preterm birth: results of a prospective study. J Am Dent Assoc 2001;132:875-80.

49. Jarjoura K, Devine PC, Perez-Delboy A, Herrera-Abreu M, D'Alton M, Papapanou P. Markers of periodontal infection and preterm birth. Am J Obstet Gynecol 2005;192:513-9.

50. Noack B, Klingenberg J, Weigelt J, Hoffman T. Periodontal status and preterm low birth weight: a case control study. J Periodontal Res 2005;40:339-45.

51. Radnai M, Gorzo I, Nagy E, Urban E, Novak T, Pal A. A possible association between preterm birth and early periodontitis: a pilot study. J Clin Periodontol 2004;31:736-41.

52. Pihlstrom BL, Michalowicz BS, Johnson NW. Periodontal diseases. Lancet 2005;366:1809-20.

53. Bascones Martínez A, Figuero Ruiz E. Periodontal diseases as bacterial infection. Av Periodon Implantol 2005;17:111-8.
54. Laine MA. Effect of pregnancy on periodontal and dental health. Acta Odontol Scand 2002;60:257-64.

55. Wimmer G, Pihlstrom BL. A critical assessment of adverse pregnancy outcome and periodontal disease. J Clin Periodontol 2008;35:380-97.

56. Àgueda A, Echeverría A, Manau C. Association between periodontitis in pregnancy and preterm or low birth weight: Review of the literature. Med Oral Patol Oral Chir Buca 2008;13:e609-15.

57. Dannan A. The periodontal disease as a risk factor for preterm birth and low birth weight: a review of case-control studies. The internet Journal of Gynecology and Obstetrics 2008 vol 10 (1).

58. Clothier B, Stringer M, Jeffcoat MK. Periodontal disease and pregnancy outcomes: exposure, risk and intervention. Best Pract Res Clin Obstet Gynaecol 2007;21:451-66. Epub 2007 Mar 23.

59. Xiong $X$, Buekens $P$, Fraser WD, Beck J, Offenbacher S. Periodontal disease and adverse pregnancy outcomes: a systematic review. BJOG 2006;113:135-43.

60. Michalowicz BS, Hodges JS, DiAngelis AJ, Lupo VR, Novak MJ, Ferguson JE, Buchanan W, Bofill J, Papapanou PN, Mitchell DA, Matseoane S, Tschida PA. Treatment of periodontal disease and the risk of preterm birth. N Engl J Med 2006;355:1885-94.

61. Goldenberg RL, Culhane JF. Preterm birth and periodontal disease. N Engl J Med 2006;355:1925-7.

62. Srinivas SK, Sammel MD, Stamilio DM, Clothier B, Jeffcoat MK, Parry S, Macones GA, Elovitz MA, Metlay J. Periodontal disease and adverse pregnancy outcomes: is there an association ? Am J Obstet Gynecol 2009;200:497.e1-8

63. Wood S, Frydman A, Cox S, Brant R, Needoba S, Eley B, Sauve R. Periodontal disease and spontaneous preterm birth: a case control study. BMC Pregnancy Childbirth 2006;6:24.

64. Skuldbøl T, Johansen KH, Dahlén G, Stoltze K, Holmstrup P. Is preterm labor associated with periodontitis in a Danish maternity ward ? J Clin Periodontol 2006;33:177-83.

65. Rajapakse PS, Nagarathne M, Chandrasekra KB, Dasanayake AP. Periodontal disease and prematurity among non-smoking Sri Lankan women. J Dent Res 2005;84:274-7.

66. Castaldi JL, Bertin MS, Giménez F, Lede R. Periodontal disease: is it a risk factor for premature labor, low birth weight or preeclampsia? Rev Panam Salud Publica 2006;19:253-8.

67. McGaw T. Periodontal disease and preterm delivery of low-birthweight infants. J Can Dent Assoc 2002;68:165-9.

68. Agueda A, Ramón JM, Manau C, Guerrero A, Echeverría JJ. Periodontal disease as a risk factor for adverse pregnancy outcomes: a prospective cohort study. J Clin Periodontol 2008;35:16-22. Epub 2007 Nov 21.

69. Abiad M. Les maladies parodontales et les naissances prématurées. JODQ 2007:12-3.

70. Polyzos NP, Polyzos IP, Mauri D, Tzioras S, Tsappi M, Cortinovis I, Casazza G. Effect of periodontal disease treatment during pregnancy on preterm birth incidence: a metaanalysis of randomized trials. Am J Obstet Gynecol. 2009;200:225-32.

71. Konopka T. Periodontitis and preterm low birth weight. Ginekol Pol 2004;75:397-403.

72. Jeffcoat M, Parry S, Sammel M, Macones G. Successful periodontal therapy reduces the risk of prematurity. AJOG 2009;201(Suppl. 6):S181.

73. Jeffcoat MK, Hauth JC, Geurs NC, Reddy MS, Cliver SP, Hodgkins PM, Goldenberg RL. Periodontal disease and preterm birth: results of a pilot intervention study. J Periodontol 2003;74:12148. 
74. Novak T, radnai M, Gorzo I, Urban E, Orvos H, Eller J, Pal A. prevention of preterm delivery with periodontal treatment. Fetal Diagn Ther 2009;25:230-3.

75. Lopez N, Smith P, Gutierrez J. Periodontal therapy may reduce the risk of preterm low birthweight in women with periodontal disease: a randomized controlled trial. J Peridontol 2002;73:911-24

76. Bisson-Boutelliez C. Desulfovibrio spp. dans la maladie parodontale : interactions avec les cellules épithéliales KB et activité de l'amoxicilline libre ou complexée sur ses formes extracellulaires et intracellulaires. Thèse de pharmacie, Faculté de Nancy 1, 2009.

77. Macones GA, Parry S, Nelson DB, Strauss JF, Ludmir J, Cohen AW, Stamilio DM, Appleby D, Clothier B, Sammel MD, Jeffcoat $M$. Treatment of localized periodontal disease in pregnancy does not reduce the occurrence of preterm birth: results from the Periodontal Infections and Prematurity Study (PIPS). Am J Obstet Gynecol 2010;202:147.e1-8.

78. Michalowicz BS, Durand R. Maternal periodontal disease and spontaneous preterm birth. Periodontology 2007;44:103-12.

79. Stamilio DM, Chang JJ, Macones GA. Periodontal disease and preterm birth: do the data have enough teeth to recommend screening and preventive treatment ? Am J Obstet Gynecol 2007; 196:93-4.

80. Yeo BK, Lim LP, Paquette DW, Williams RC. Periodontal Disease - The emergence of a risk for systemic conditions: pre-term low birth weight. Ann Acad Med Singapore 2005;34:111-6.

81. Ovadia R, Zirdok R, Diaz-Romero RM. Relationship between pregnancy and periodontal disease. Med Biol 2007;14:10-4.

82. Li X, Kolltveit KM, Tronstad L, Olsen I. Systemic diseases caused by oral infection. Clin Microbiol Rev 2000;13:547-58.
83. Offenbacher S, Beck JD, Lieff S, Slade G. Role of periodontitis in systemic health: spontaneous preterm birth. J Dent Educ 1998;62:852-8

84. Boggess KA, Moss K, Madianos P, Murtha AP, Beck J, Offenbacher $S$. Fetal immune response to oral pathogens and risk of preterm birth. Am J Obstet Gynecol 2005;193:1121-6.

85. Task Force on Periodontal Treatment of Pregnant Women, American Academy of Periodontology. American academy of periodontology statement. Regarding periodontal management of the pregnant patient. J Periodontol 2004;75:495.

86. Periodontal disease may pose one risk for premature birth. J Am Med Assoc website: http://jama.ama-assn.org/issues/v283n22/ ffull/jmn0614-4.html. Accessed 5 May 2010.

87. New York State Department of Health. Oral health care during pregnancy and early childhood - practice guidelines. August 2006.

88. Van De Geyn L. Attention à la bouche (pp. 27-9). Santé canadienne, novembre-décembre, 2008.

89. Phaneuf P, Parent C. La parodontie et l'obstétrique. Cours de formation continue. Université de Laval (Canada) 2008

90. Hamissi J, BakianianVaziri P, Davalloo A. Evaluating oral hygiene knowledge and attitude of pregnant women. Iranian J Publ Health 2010;39:28-31.

91. Noochpoung R, Dejpitak A, Yoshitoku Y, Junichi S. Dental caries and gingivitis among pregnant and non-pregnant women in Chiang Mai, Thailand. Nagoya J Med Sci 2010;72:43-50.

92. Boggess KA. Maternal oral health in pregnancy. Obstet Gynecol 2008;111:976-86.

93. Betleja-Gromaldi K, Janicka K, Ronin-Walknowska E, Banach J, Mazurek-Mochol M. Periodontitis and the premature labor in the light of the clinical examinations in the selected population of women. Archi Perinat Med 2007;13:49-54. 